\title{
Apport des datations U/Th de spéléothèmes à la connaissance de l'incision du réseau hydrographique de l'Est du Bassin parisien
}

Contribution of the U/Th datings of speleothems to knowledge of the incision of the hydrographic network, eastern France Beitrag höhlenkundlicher Datierungen zur Kenntnis des Einschneidens des hydrographischen Netzes im Osten Frankreichs

Stéphane Jaillet, Benoît Losson, Jacques Brulhet, Jeannine Corbonnois, Bruno Hamelin, Edwige Pons-Branchu et Yves Quinif

\section{OpenEdition}

Journals

Édition électronique

URL : http://journals.openedition.org/rge/2689

DOI : 10.4000/rge.2689

ISSN : 2108-6478

Éditeur

Association des géographes de l'Est

Édition imprimée

Date de publication : 1 septembre 2002

ISSN : 0035-3213

\section{Référence électronique}

Stéphane Jaillet, Benoît Losson, Jacques Brulhet, Jeannine Corbonnois, Bruno Hamelin, Edwige PonsBranchu et Yves Quinif, « Apport des datations U/Th de spéléothèmes à la connaissance de l'incision du réseau hydrographique de l'Est du Bassin parisien », Revue Géographique de l'Est [En ligne], vol. 42 / 4 | 2002, mis en ligne le 17 janvier 2011, consulté le 10 décembre 2020. URL : http:// journals.openedition.org/rge/2689; DOI : https://doi.org/10.4000/rge.2689

Ce document a été généré automatiquement le 10 décembre 2020.

Tous droits réservés 


\title{
Apport des datations U/Th de spéléothèmes à la connaissance de l'incision du réseau hydrographique de l'Est du Bassin parisien
}

\author{
Contribution of the U/Th datings of speleothems to knowledge of the incision of \\ the hydrographic network, eastern France \\ Beitrag höhlenkundlicher Datierungen zur Kenntnis des Einschneidens des \\ hydrographischen Netzes im Osten Frankreichs
}

Stéphane Jaillet, Benoît Losson, Jacques Brulhet, Jeannine Corbonnois, Bruno Hamelin, Edwige Pons-Branchu et Yves Quinif

La Lorraine et la Champagne orientale offrent des paysages de plateaux calcaires aux reliefs modérés comparés aux massifs karstiques du pourtour des Alpes, des Causses ou des Pyrénées. Aussi les études karstologiques y ont été relativement tardives dans le champ des études menées sur le karst depuis une trentaine d'années. Des travaux préliminaires (Beaudoin, 1974, 1989 ; Gamez, 1977) avaient pourtant montré l'intérêt de ce secteur de bas-plateaux, mais ce n'est qu'avec la thèse de Patrice Gamez (1992) que la première véritable étude de karstologie voit le jour en Lorraine. Essentiellement axée sur l'hydrologie et le fonctionnement du karst (secteur de la Woëvre septentrionale), elle n'offre pas d'éléments de datation à partir de dépôts sédimentologiques en grotte. Ce n'est qu'à la fin des années 1990, avec les travaux de Jaillet (2000), Pons-Branchu (2001) et Losson (en cours) ${ }^{1}$, que les premières datations U/Th sont entreprises sur les karsts de Lorraine. Les recherches menées autour du laboratoire de recherche souterrain de Meuse/Haute-Marne de l'ANDRA ${ }^{2}$ ont permis la réalisation de la plupart de ces datations. Elles ont pour vocation de caler chronologiquement les schémas d'évolution du relief, en particulier l'incision du réseau hydrographique et le recul des couvertures. L'objectif du présent article est d'intégrer la mise en place des drains majeurs du karst dans la dynamique de l'incision des vallées et du recul des couvertures. Une synthèse de l'ensemble des datations U/Th qui ont été réalisées sur 
cette région est ensuite proposée. Celles-ci sont d'un apport novateur à la connaissance de l'évolution du relief. Pour cela trois grandes vallées de la France de l'Est (la Moselle, la Meuse et la Marne) constituent le support spatial (fig. 1). Elles recoupent toutes les auréoles carbonatées de l'Est du Bassin parisien et ont permis le développement plus ou moins marqué de ces karsts.

Figure 1 : Localisation des karsts étudiés dans le contexte géologique et hydrographique de l'Est du bassin de Paris

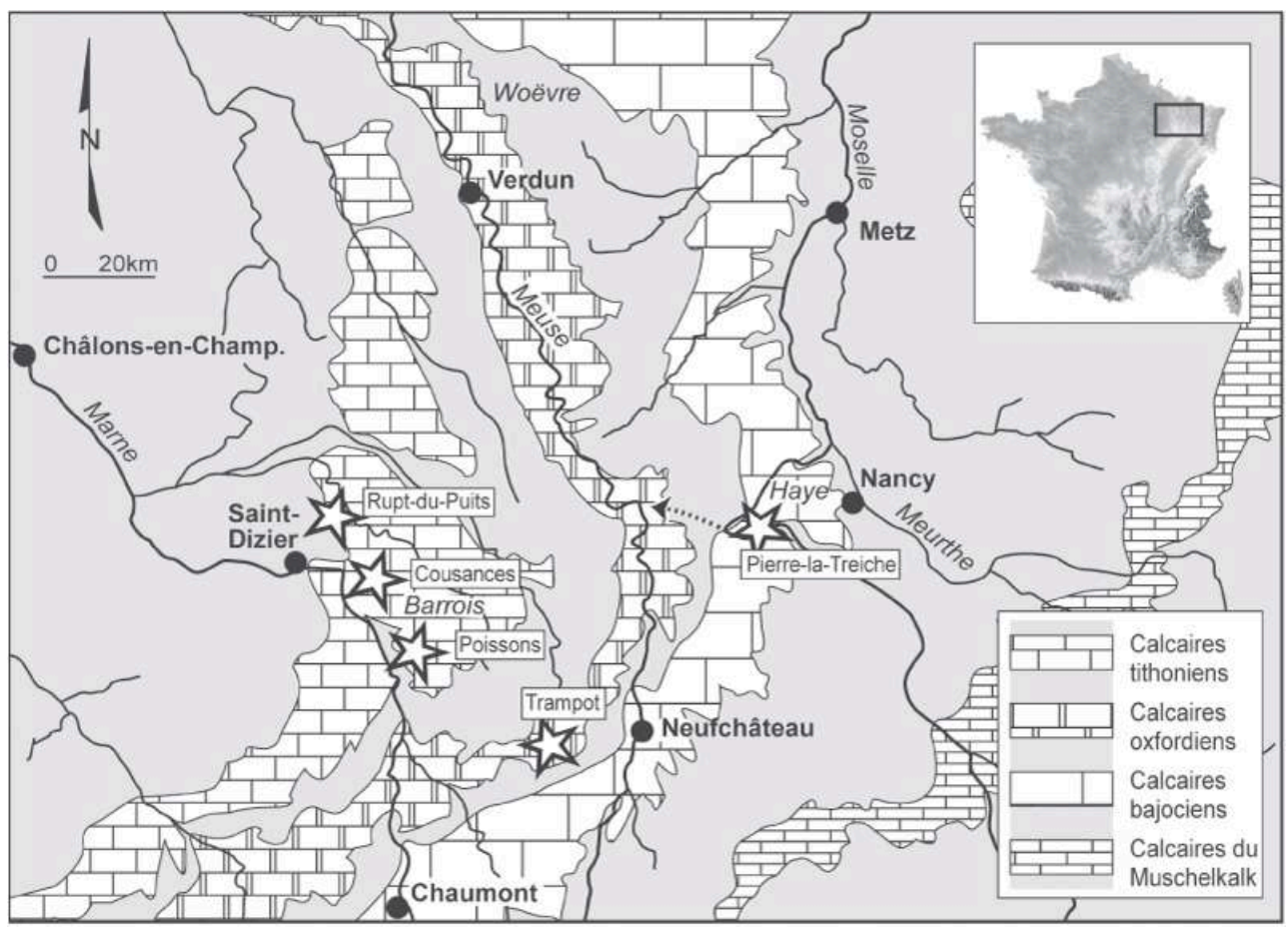

\section{La mise en place des drains du karst et leur relation Avec l'incision des vallées}

2 Parmi tous les conduits creusés dans le karst, seuls quelques drains sont à même d'enregistrer des informations exploitables pour les reconstitutions environnementales. Ces drains (généralement les plus gros) témoignent de la structuration, de la hiérarchisation et du transit de sédiments externes (matériel fluviatile) dans le karst. Sous certaines conditions, ils sont le siège de précipitation de calcite avec mise en place de spéléothèmes. Un certain nombre de ces drains du karst sont visitables spéléologiquement et ces informations (alluvions, spéléothèmes) y sont relevées. Les études menées sur l'endokarst sont cependant limitées par la taille des conduits imposant une vision parcellaire du karst. Conscient de ces limites, nous avons réalisé un grand nombre de prélèvements dans les cavités lorraines.

Dans l'Est du Bassin parisien, deux dispositifs majeurs sont identifiés pour la mise en place des drains du karst: soit au pourtour des couvertures subsistantes sur les plateaux, soit au droit des grandes vallées, sous le talweg. 


\section{A. Une karstification des plateaux en limite de couverture}

C'est le cas le plus général. Il a été reconnu par Beaudoin (1974) sur le Barrois, par Gamez (1977) en Woëvre septentrionale, par Durup de Baleine (1989) en forêt de Hesse, par Jaillet et Gamez (1995) en forêt de Trois Fontaines. La karstification des calcaires s'effectue en limite de couverture généralement argilo-sableuse. Les écoulements de surface ruissellent sur cette couverture et, une fois concentrés, rejoignent les pertes au contact de la dalle calcaire (fig. 2). De même, les niveaux sableux de la couverture constituent des réservoirs perchés qui contribuent à l'étalement du flux hydrique sur l'année hydrologique. La compresse humide (Gamez, 1992) joue donc ce rôle double sur la karstification et constitue le facteur essentiel de la localisation des karsts en Lorraine. Le recul du front de la couverture au cours du temps déplace le point de concentration des écoulements dans les calcaires et génère une migration des appareils karstiques. En outre, de part et d'autres des vallées, les drains du karst s'étagent en suivant peu ou prou cette incision (fig. 2). Ainsi, les drains les plus anciens seront les plus haut perchés tandis que les plus récents seront proches du niveau de base (Delannoy, 1997 ; Audra, 2001). Cependant, cette tendance générale doit être nuancée par le remblaiement fini-quaternaire des vallées (Carcaud, 1990 ; Weisrock, 1993), qui peut conduire à des réajustements locaux des drains karstiques. Dans les vallées de la Marne et de la Saulx, ce remblaiement alluvial génère localement la remontée du niveau des drains karstiques, mais dans un espace proche de la zone des exutoires (fig. 3).

Figure 2 : Mise en place des drains du karst associés à l'incision des vallées et au recul des couvertures non carbonatées

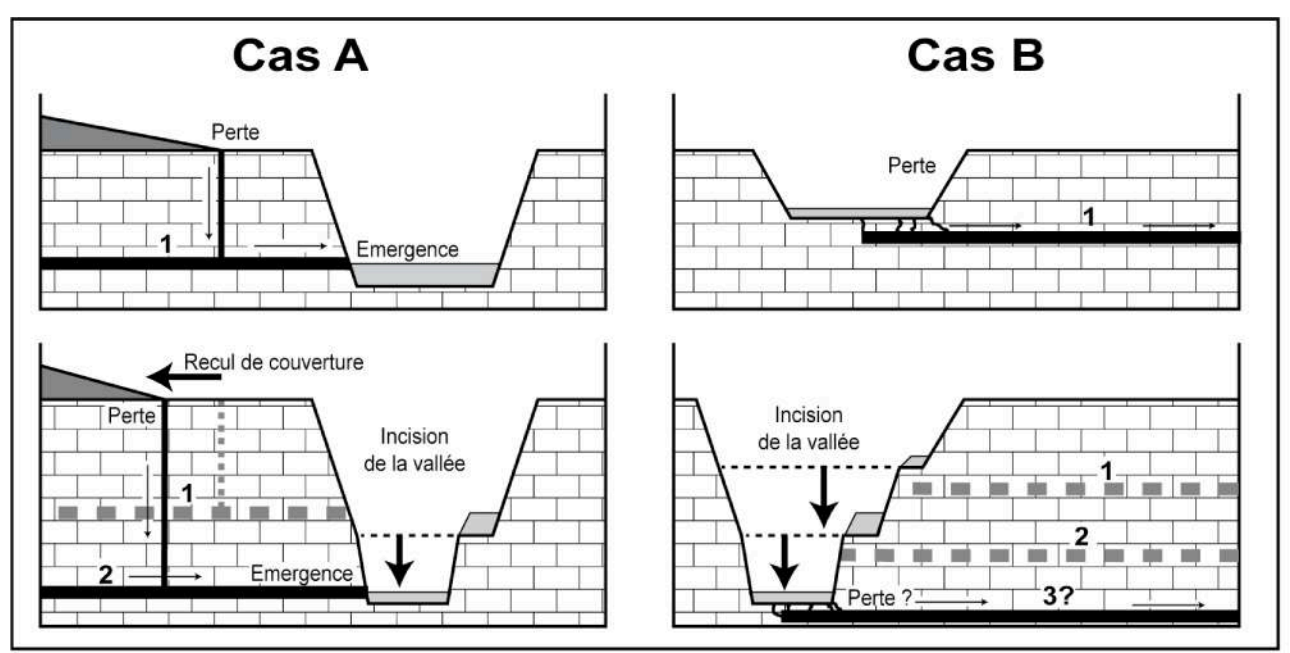


Figure 3 : Bloc-diagramme illustrant le raccordement du karst actuel au remblaiement finiquaternaire des fonds de vallées lorraines. Schéma réalisé à partir de l'exemple de la vallée de la Saulx dans sa partie aval

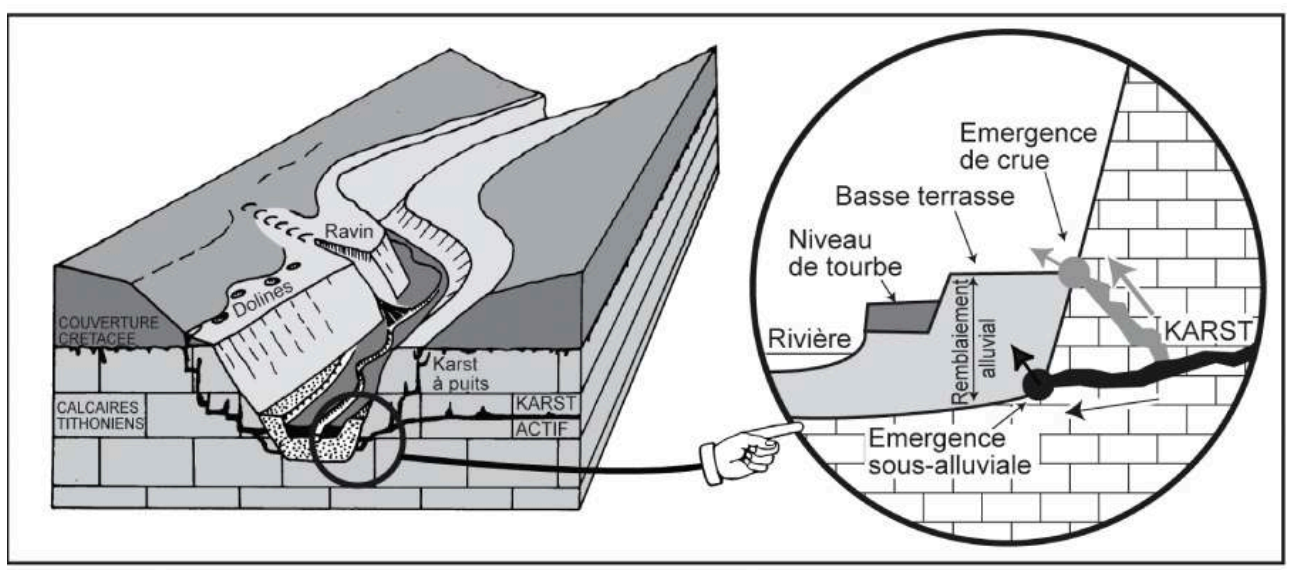

\section{B. Une karstification sous formation alluviale de fond de vallée}

Les grandes vallées lorraines drainées par les cours d'eau nés dans le Massif Vosgien (Paléo-Moselle-Meuse, Paléo-Meurthe, Moselle-et-Meurthe), recèlent d'importantes quantités de matériaux fluviatiles, essentiellement constituées d'alluvions siliceuses, issues du socle cristallin ou des terrains gréseux du Trias. Ces formations superficielles présentent actuellement en fond de vallées, une épaisseur moyenne de 5 à $10 \mathrm{~m}$ (Carcaud, 1990 ; Buzzi et al., 1993 ; Weisrock, 1993). Elles se comportent donc comme une couche géologique sablo-conglomératique meuble, dont la répartition spatiale linéaire est limitée aux tracés et paléotracés des artères hydrographiques majeures.

La surimposition de celles-ci à travers les systèmes de cuestas jurassiques (Le Roux et Harmand, 1998), implique le recoupement de massifs à dominante carbonatée (revers calcaires du Muschelkalk, du Bajocien et de l'oxfordien). On trouve alors une situation analogue aux plateaux sous couverture, c'est-à-dire le développement d'un karst, se développant sous la vallée, en relation avec une formation sus-jacente (la masse alluviale), qui joue un rôle de compresse humide (fig. 2). De toutes les conditions nécessaires à l'endokarstification (Quinif, 1998), deux sont ubiquistes : substratum fracturé et nappe phréatique alluviale dont l'eau agressive est constamment renouvelée par échanges hydrologiques avec les rivières. La dernière, constituée par le gradient hydraulique, est moins généralisée dans l'espace; elle est fonction des contextes géomorphologiques propres à chaque tronçon de vallée (largeur, pente).

7 La différence principale entre les karsts de plateaux sous couverture et les karsts sousfluviaux tient donc au gradient hydraulique, bien plus faible dans le second cas. Une certaine compensation résulte des quantités d'eau, plus importantes dans les vallées, grâce aux rivières allochtones.

\section{Relation entre ces deux dispositifs et l'incision des vallées}

Dans un cas comme dans l'autre, l'enfoncement du réseau hydrographique conduit à des réajustements des drains du karst (fig. 2). Ces réajustements s'expriment par le jeu d'un étagement souterrain des conduits. À chaque stade d'incision va ainsi 
correspondre un nouvel épisode de karstification associé à la mise en place d'une nouvelle série de drains. Les drains les plus hauts perchés sont abandonnés au profit de niveaux d'écoulement plus profonds en relation avec le nouveau niveau de base que constitue la vallée. Dans le cas des karsts de plateaux sous couverture (fig. 2 - cas A), le niveau des drains est globalement calé au droit de la surface piézomètrique et donc au niveau de la rivière (à moins de 5 mètres près). A l'inverse dans le cas des karsts sous fluviaux (fig. 2 - cas B), la karstification s'opère sous le niveau de la vallée (parfois sur plus de 10 mètres de profondeur). C'est dire que l'étude d'un drain de ce karst sera à rattacher à un niveau de terrasses nettement supérieur à l'altitude du conduit analysé. Cette différence (cas A/cas B) impose donc une reconnaissance fine des morphologies souterraines afin de distinguer précisément dans quel dispositif morphogénétique se situe le conduit étudié. La nature des remplissages, les morphologies des parois (en particulier les paléosens de circulation) permettent généralement de trancher sans ambiguité. Dans la suite, nous présentons deux exemples de karsts de plateaux sous couverture et un cas de karst sous-fluvial.

Dans l'Est du Bassin parisien, les épisodes d'incision ont, en outre, été clairement identifiés dans les vallées de la Moselle, de la Meuse (Harmand, 1992) et de la Marne. Cette dernière, avec ses affluents de rive droite, la Saulx et l'Ornain, a fait l'objet de travaux récents conduisant à un découpage de l'incision en 4 stades notés « $\mathrm{D}$ », « $\mathrm{C}$ », « B », « A » (Harmand et al., 2002; Marre et al., 2002). Il est possible de raccorder certaines phases de karstification du plateau tithonien à ces stades d'incision.

\section{Quelques karsts de l'Est du Bassin parisien associés à de grandes vallées}

10 A travers les exemples de la Marne, de la Meuse et de la Moselle, trois axes de drainage majeurs recoupant respectivement les auréoles calcaires du Bajocien, de l'oxfordien et du Tithonien, on montre que la karstification est intimement liée à l'incision des vallées dans les masses carbonatées.

\section{A. Le karst sous-fluvial des calcaires bajociens de la Moselle}

11 Les réseaux karstiques que l'on rencontre à flanc de versant de la vallée cataclinale de la Moselle, dans le plateau de Haye, revers de la cuesta «bajocienne », (Gamez et al., 1995), résultent d'une spéléogenèse liée à des pertes de la rivière sous le talweg, comme le prouvent divers éléments morphologiques et sédimentologiques hypogés (Gamez et Losson, 1998; Losson, 2000, 2001). Ces mêmes indices ont permis de préciser la profondeur minimale à laquelle se sont développées les galeries, sous le fond de la vallée. A Pierre- la-Treiche, l'essentiel des cavernes a été créé avant l'encaissement de la Moselle dans ses alluvions F4, que l'on retrouve en grande quantité sous terre, jusqu'à $-25 \mathrm{~m}$ sous le talweg rocheux de la formation alluviale considérée (fig. 4). Le détournement de la Haute Moselle vers le bassin versant rhénan s'est produit précisément à la fin du remblaiement de la formation F4 (noté Fx2 in Harmand et al., 1995 ; Pissart et al., 1997). 
Figure 4 : Le karst de Pierre-la-Treiche (calcaires bajociens) est constitué d'anciennes pertes sousfluviales de la Moselle aujourd'hui étagées et dont les remplissages alluviaux sont scellés par des spéléothèmes

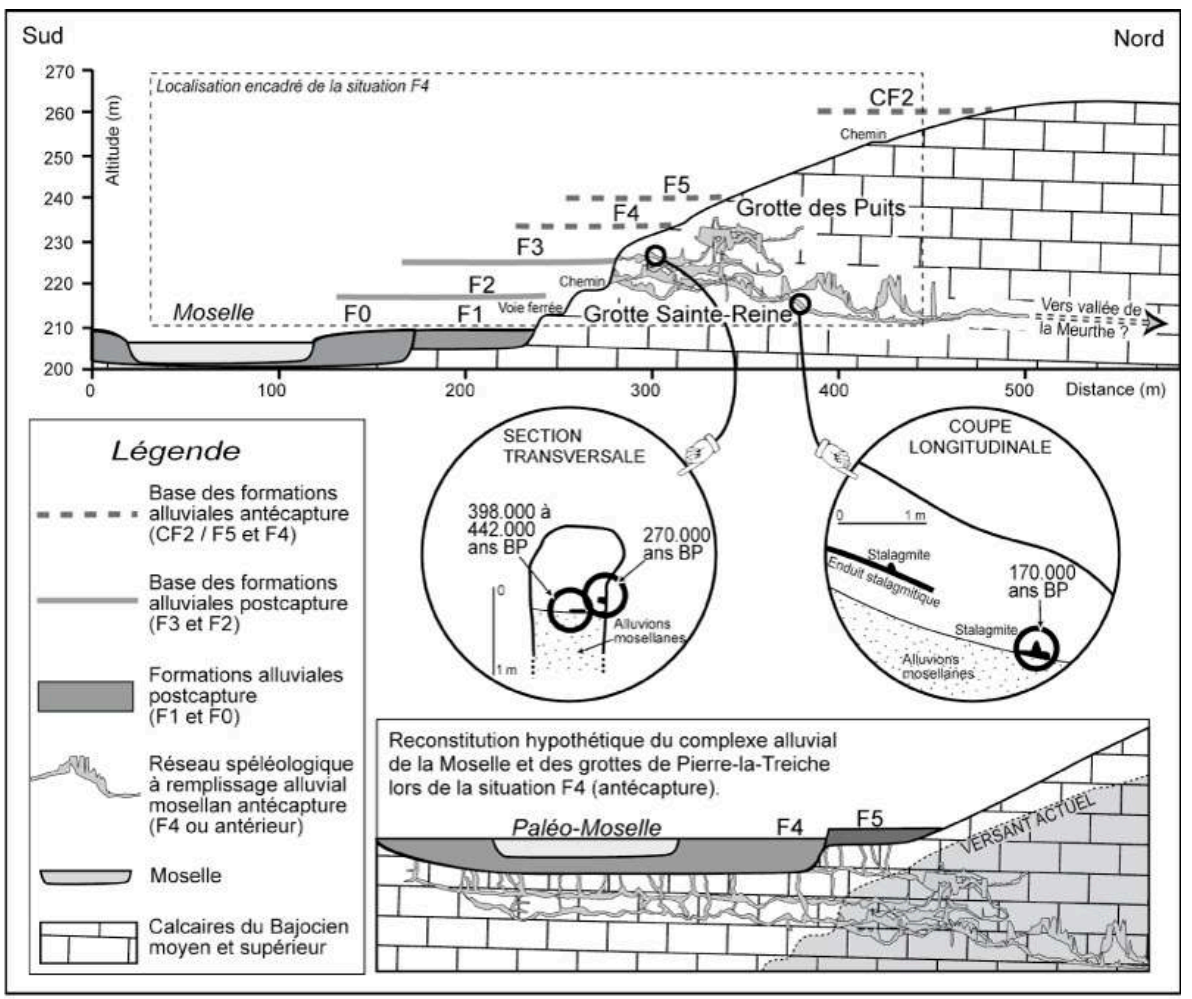

12 Cette dernière considération est primordiale pour comprendre les raisons de la karstification sous-fluviale, particulièrement exprimée le long du cours cataclinal de la Moselle, et à une telle profondeur. En effet, hormis une phase initiale probable en régime noyé, des écoulements libres ont participé à l'évolution spéléologique des réseaux, ainsi qu'on a pu le déceler dans les remplissages endokarstiques attribués à la formation F4. Un tel fonctionnement hydrogéologique ne peut s'expliquer que par l'existence d'un secteur déprimé pouvant recueillir les infiltrations mosellanes au sein du plateau de Haye. Ce niveau de base correspond nécessairement au bassin versant de la Meurthe, qui commandait le degré de structuration de l'endokarst, et donc la pente de la surface piézométrique (Quinif, 1994).

Le contexte géomorphologique précédant la capture de la Moselle, avec deux bassins versants situés à des altitudes moyennes différentes, est donc pleinement responsable de la majeure partie de la karstification reconnue du plateau central de Haye, ceint actuellement par la «Boucle de la Moselle» (fig. 1). La présence de petites cavités d'âge postcapture dans la paléovallée du Terrouin, désormais empruntée par la Moselle, à l'aval de Toul, indique la persistance d'infiltrations sous-fluviales après le détournement hydrographique et une adaptation au nouveau contexte géomorphologique.

Ainsi, le karst sous fluvial des calcaires bajociens du plateau de Haye s'est-il mis en place au cours de l'incision de la vallée de la Moselle. La plupart des cavités reconnues traduisent une phase de karstification antérieure à la capture de la Moselle et l'étude des dépôts de ces cavités renseigne sur les conditions paléo-environnementales qui prévalait au moment de leur abandon (phase de remplissage). C'est-à-dire que l'analyse 
de spéléothèmes scellant ces dépôts de grottes permet de dater à minima la capture de la Moselle (Losson et Quinif, 2001).

\section{B. Le karst des calcaires oxfordiens et de la haute vallée de la Meuse}

15 «Des grands massifs calcaires lorrains, l'oxfordien fait figure de parent pauvre de la karstologie, à l'exception de la région de Neufchâteau »(Gamez, 1985). En effet, la karstification de l'oxfordien est lacunaire et c'est vers le haut bassin de la Meuse, dans le secteur de Trampot, au droit de la ligne de partage des eaux de la Marne, de l'Ornain et de la Meuse, que l'on rencontre les phénomènes les plus spectaculaires. Ici, dans une unité karstique de $15 \mathrm{~km} 2$ environ, une centaine de phénomènes karstiques a été répertoriée. Les Calcaires à Astartes inférieurs font ici office de couverture plus ou moins argileuse.

16 Vingt mètres sous la surface, le réseau Hadès (fig. 5) est un tube horizontal syngénétique, tracé en baïonnette, de 2 à $5 \mathrm{~m}$ de diamètre, marquant un ancien niveau phréatique (Jaillet, 2000). Le réseau a été ensuite abandonné, ce que montrent les puits trépanant les galeries à l'emporte-pièce, la détente générale du réseau, les éboulis, le basculement des banquettes pariétales et l'écartement des parois. Ces éléments sont la marque de l'abandon progressif de cette paléostructure de drainage endokarstique associé à une détente lithostatique généralisée.

17 Nous proposons de raccorder cette décompression généralisée au décapage en surface d'une tranche de couverture. La détente lithostatique superficielle, ajoutée au soutirage des écoulements, déstructure le réseau souterrain et fragilise les voûtes. Certaines parois s'écartent et génèrent la rupture de planchers stalagmitiques datables. 
Figure 5 : Le réseau Hadès (calcaires oxfordiens) témoigne d'un haut niveau perché des écoulements karstiques et d'une ancienne extension de la couverture du Séquanien, aujourd'hui démantelée

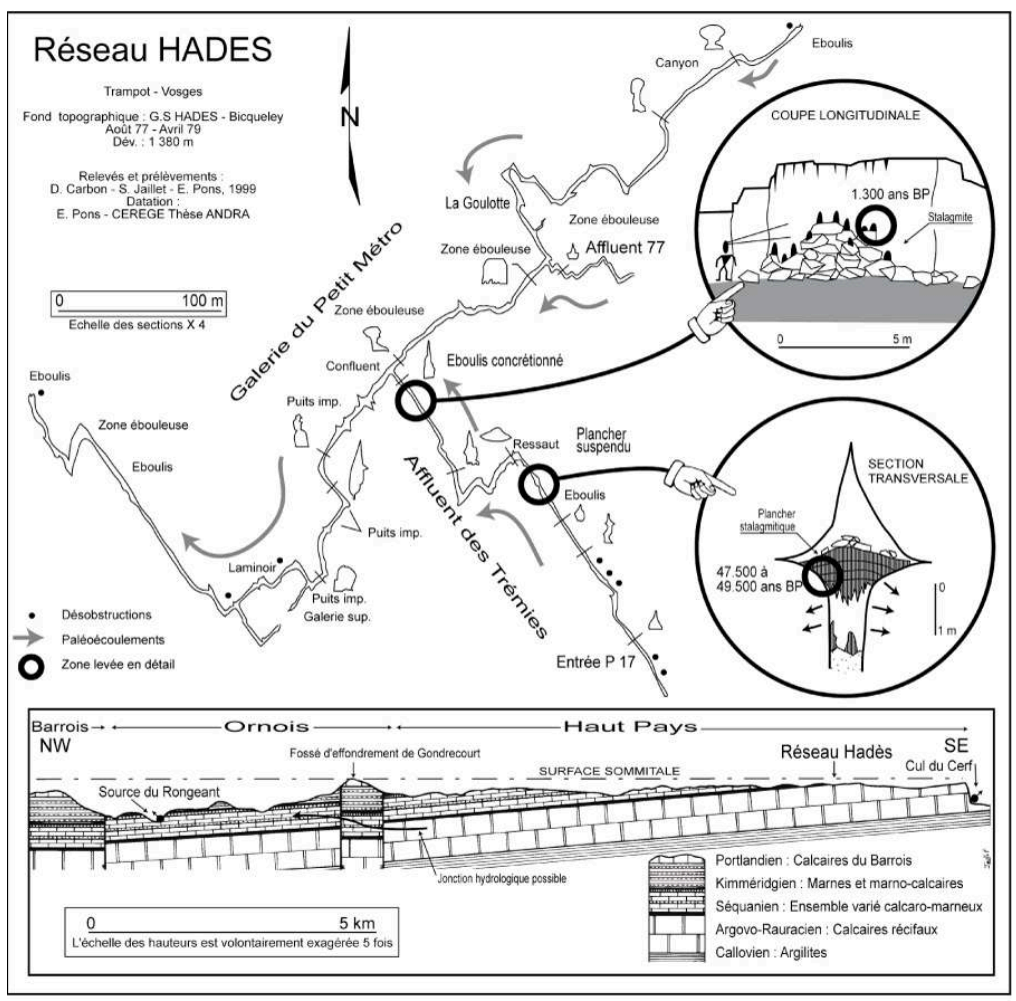

18 Il est par contre délicat de rattacher clairement le tube phréatique originel du réseau Hadés à un stade d'incision du réseau hydrographique de l'Est du Bassin parisien. Ce secteur géographique est perché à $400 \mathrm{~m}$ d'altitude, dans les têtes de réseau hydrographique de la Marne et de la Meuse. Les écoulements souterrains actuels semblent d'ailleurs divergents et il est possible qu'une partie de l'aquifère des calcaires oxfordiens se vidange dans la vallée de la Marne par un de ses affluents, le Rongeant (coupe fig. 5 ; Jaillet, 2000). On ne cherchera donc pas ici à rattacher ce réseau à un stade d'évolution de la vallée de la Meuse ou de la Marne. Il est par contre très intéressant de relier son évolution à la dynamique du retrait de la couverture des Calcaires à Astartes inférieurs. C'est ce que l'étude et la datation des spéléothèmes vont permettre.

\section{Le karst des calcaires tithoniens de la vallée de la Marne}

19 Le massif de Poissons (fig. 6) est aujourd'hui un karst à puits comblés, exploité en minières au cours du XIX ${ }^{e}$ siècle (Cornuel, 1879). C'est une unité discontinue, mais longiligne, de 1 à $3 \mathrm{~km}$ d'est en ouest et de 8 à $10 \mathrm{~km}$ du nord au sud, qui jalonne l'ancien contact de la couverture crétacée avec les calcaires tithoniens sous-jacents, dans un espace délimité entre les vallées de la Marne et de la Saulx (Jaillet, 2000). La synthèse des descriptions de ces minières et leur cartographie laissent penser que le paléo-niveau des écoulements horizontaux de ce karst devait se situer vers 300 à $330 \mathrm{~m}$ d'altitude, soit environ, de 120 à $150 \mathrm{~m}$ au-dessus des écoulements actuels de la Marne. Il se raccorde donc aux épisodes anciens de l'incision du réseau hydrographique local (épisode « D » in Harmand et al., 2002 ; Marre et al., 2002). Les karsts actifs, tels ceux 
de Savonnières-en-Perthois ou du Rupt du Puits, sont plus récents. Ce dernier, d'un développement spéléologique supérieur à $10 \mathrm{~km}$, constitue à ce jour le réseau le plus important du Bassin parisien. C'est un exemple remarquable de karstification récente où l'organisation est déjà très aboutie. La position géographique de ces réseaux souterrains implique un déblaiement d'une épaisseur de 70 à $100 \mathrm{~m}$ de Crétacé avant que la table calcaire tithonienne ne soit atteinte. Aussi leur existence est-elle plutôt liée aux dernières incisions des vallées du Barrois et de la Marne en particulier (épisodes « $\mathrm{B}$ » et « $\mathrm{A}$ » d'incision du réseau hydrographique; Harmand et al., 2002; Marre et al., 2002).

Figure 6 : Le karst de Poissons (calcaires tithoniens) est le témoin d'une ancienne extension de la couverture crétacée et d'un niveau de la Marne perché de 120 à 150 m au-dessus des écoulements actuels

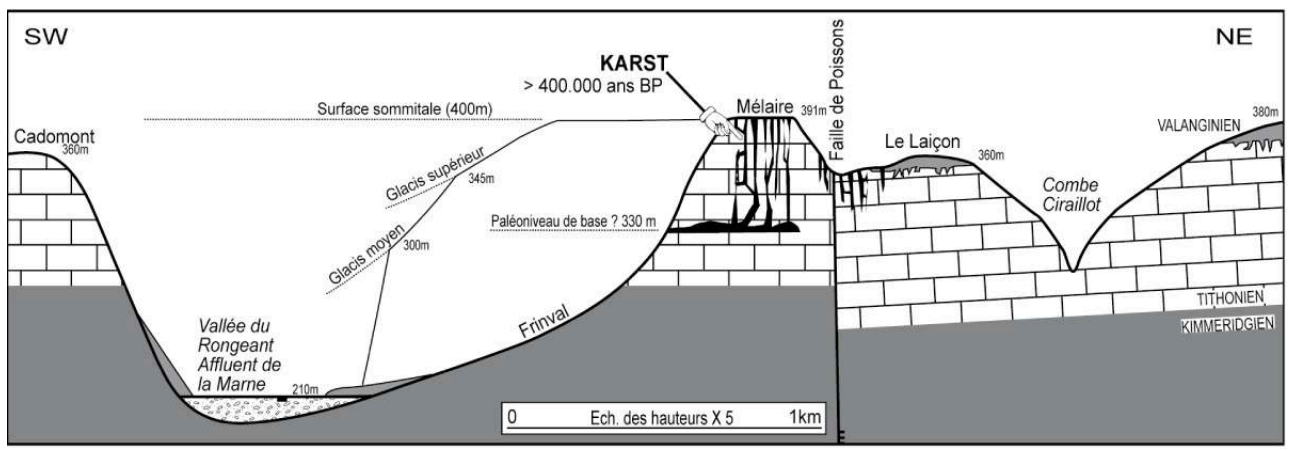

\section{Les datations U/Th}

84 datations (dont 73 exploitables) ont été réalisées dans six ensembles spéléologiques de l'Est du Bassin de Paris (tabl. 1). Elles sont toutes rassemblées pour la première fois dans cette synthèse (fig. 7). Elles ont été réalisées au CERAK (Centre d'Études et de Recherches Appliquées au Karst) de Mons, en Belgique, par la méthode dite de spectrométrie ?3 (36 datations ; Quinif, 1989 ; Jaillet, 2000 ; Losson, thèse en cours) ou au CEREGE (Centre Européen de Recherche et d'Enseignement en Géoscience de l'Environnement) à Aix-en-Provence par la méthode $\mathrm{TIMS}^{4}$ (48 datations ; PonsBranchu, 2001). Elles couvrent l'ensemble de la méthode U/Th (0 à 350000 ans). Ces dates ont été obtenues lors de campagnes de prélèvements définies autour de problématiques géomorphologiques et où le contexte a été reconnu afin d'assurer un contrôle correct (position du spéléothème dans la cavité et dans le milieu karstique, enchaînement des séquences sédimentaires endokarstiques...). 
Tableau 1 : Présentation des 84 datations U/Th réalisées sur 6 systèmes karstiques de l'Est du Bassin parisien

\begin{tabular}{|l|c|c|c|c|c|}
\hline \multirow{2}{*}{ Etage } & $\begin{array}{c}\text { Systèmes } \\
\text { karstiques }\end{array}$ & $\begin{array}{c}\text { Nb. datations } \\
\text { méthode } \alpha\end{array}$ & $\begin{array}{c}\text { Nb. datations } \\
\text { méthode } \\
\text { TIMS }\end{array}$ & $\begin{array}{c}\text { Total } \\
\text { datations }\end{array}$ & Gamme de datation \\
\hline \multirow{3}{*}{ Tithonien } & Rupt-du-Puits & 2 & 24 & 26 & 0 à 32000 ans BP \\
\cline { 2 - 7 } & Cousances & 4 & 10 & 14 & 16000 à 150000 ans BP \\
\cline { 2 - 7 } & Poissons & 4 & 0 & 4 & 29000 à + de 400000 ans \\
\hline Oxfordien & Hadès & 0 & 4 & 4 & 1000 à 50000 ans BP \\
\hline \multirow{3}{*}{ Bajocien } & Pierre-la-Treiche & 24 & 10 & 34 & 0 à + de 350000 ans BP \\
\cline { 2 - 7 } & Marron & 2 & 0 & 2 & 55000 à 70000 ans BP \\
\hline
\end{tabular}

Figure 7 : Classement des datations U/Th obtenues sur les karsts lorrains (Maron, Pierre-laTreiche, Hadès, Poissons, Cousances, Rupt-du-Puits) en regard de la courbe 㜯 $18 \mathrm{O}_{\text {atm }}$ du forage de Vostok en Antarctique (Petit et al., 1999)

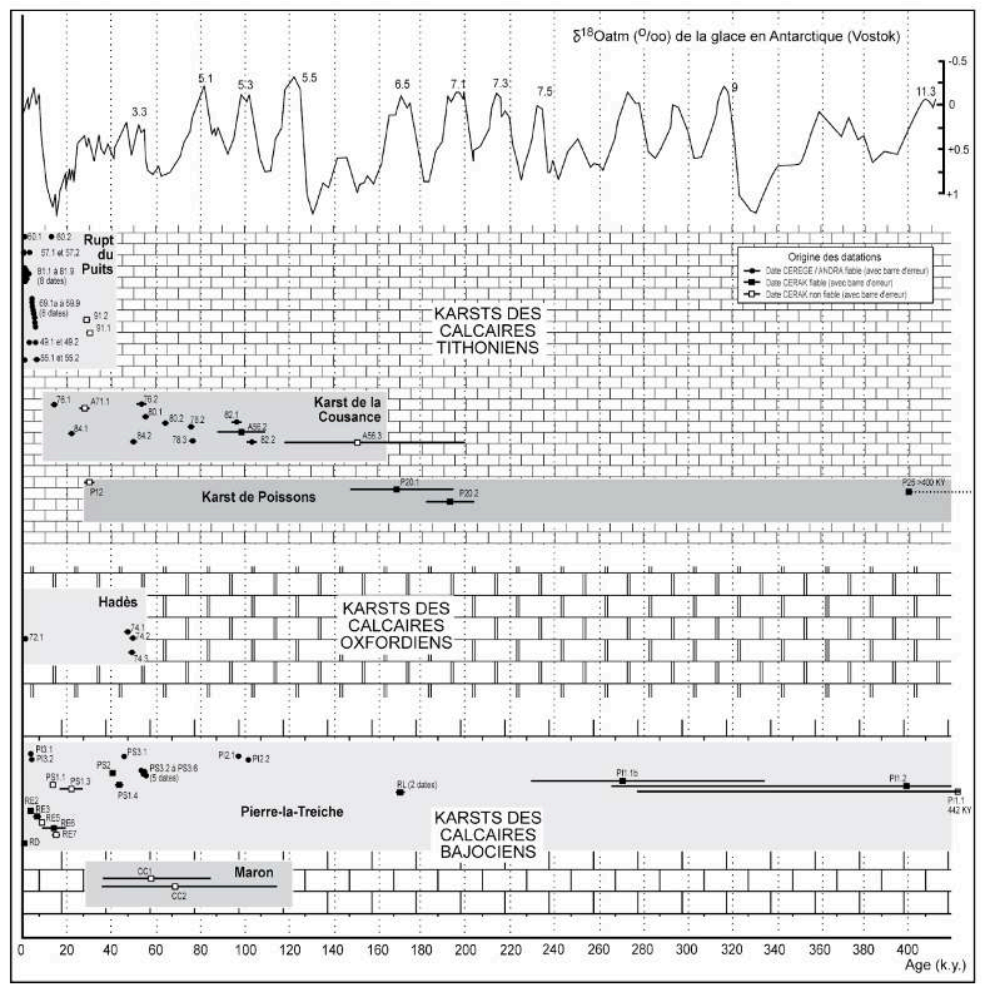

\section{A. Les résultats}

Dans le karst du plateau de Haye, les prélèvements ont été effectués dans les grottes Sainte-Reine et des Puits. Elles appartiennent toutes deux aux systèmes des grottes de Pierre-la-Treiche. Des analyses ont aussi été effectués dans la grotte de la carrière à Marron, mais les résultats les plus intéressants et les plus fiables ont été obtenus dans les cavités de Pierre-la-Treiche. Les dates s'étalent sur l'ensemble de la méthode ( 0 à 350000 ans) avec une forte proportion dans les stades isotopiques 3 et 1 (fig. 7). Les plus anciennes $(170000,270000$ et plus de 350000 ans B.P.) ont été obtenues dans les réseaux spéléologiques les plus bas en altitude. Les observations morphologiques et l'analyse des sédiments endokarstiques montrent que tous ces 
réseaux se raccordent à un épisode anté-capture de la Moselle. La large gamme de dates obtenue sur cet ensemble montre l'absence de relation absolue entre l'âge des conduits karstiques et la pousse des concrétions qui suit la mise en place de ces conduits. Seuls les datations les plus anciennes seront réellement exploitables dans la reconstitution de l'évolution géomorphologique, en particulier de l'incision du réseau hydrographique.

Dans le karst de Trampot (réseau Hadès), un plancher reposant sur deux banquettes de la galerie syngénétique originelle a été daté entre 47500 et 49500 ans B.P. (PonsBranchu, 2001). Ce plancher a subi une phase de décompression généralisée liée à la détente du massif (fig. 5). Dans le même réseau, un éboulis lié à cette détente est scellé par une dizaine de petites stalagmites dont l'une a pu être datée de 1260 ans B.P. (PonsBranchu, 2001). Les datations entreprises sur ce réseau laissent penser que cette détente généralisée n'a pu se produire qu'après le stade isotopique 3, probablement pendant le dernier maximum glaciaire (stade isotopique 2).

Dans les karsts du Barrois, les prélèvements ont été effectués à Poissons, à Cousances et au Rupt-du-Puits. Dans le plus haut perché de ces karsts (Poissons), une datation dépasse les limites de la méthode (350 à 400000 ans B.P.) et montre qu'au cours du Pléistocène moyen, ce karst était déjà en voie de comblement (Jaillet, 2000). Un autre échantillon, daté de 168800 à 194000 ans B.P., montre qu'à ce moment-là, des écoulements susceptibles de générer la précipitation de calcite transitaient encore dans ce karst. Ce n'est plus le cas aujourd'hui, où l'ensemble des conduits est comblé d'un remplissage ferrugineux issu du démantèlement du Crétacé. Bien qu'on n'accède plus aujourd'hui qu'à la partie superficielle du karst, on suppose que sa mise en place a été similaire à celle des karsts actifs reconnus aujourd'hui plus au nord (Jaillet, 2000).

Dans le karst de Cousances, des prélèvements ont été effectués à différents niveaux des réseaux, jusqu'à des conduits perchés à une quinzaine de mètres au-dessus de la Marne. Les datations les plus anciennes (100 000 à 150000 ans B.P.) impliquent qu'au cours de l'Eémien (stade isotopique 5), le niveau des écoulements de la Marne devait se situer au plus à une dizaine de mètres au-dessus des écoulements actuels (Pons-Branchu, 2001; Jaillet et al., soumis). Dans le karst du Rupt-du-Puits, toutes les datations appartiennent aux stades isotopiques 1 et 3 , raccordés à un fonctionnement récent de ce système karstique.

\section{B. Le raccordement de ces datations au contexte géomorphologique}

Avant d'intégrer ces résultats dans le cadre de l'évolution géomorphologique de l'Est du Bassin parisien, il convient de s'interroger sur la signification réelle de ces datations U/ Th de spéléothèmes et sur leur raccordement à l'évolution du relief. Les concrétions de grottes croissent dans un contexte bien particulier. La zone karstique doit être non saturée, c'est-à-dire que le conduit doit être abandonné de tout écoulement phréatique ou fluviatile. Certaines concrétions poussent en zone épinoyée, mais des critères morphoscopiques (corrasion, intercalations détritiques), permettent généralement de les reconnaître. Le spéléothème pousse alors et scelle un conduit et/ou un remplissage. Outre les problèmes liés à l'analyse géochimique de l'échantillon et les barres d'incertitudes liés à ces analyses, il est bon de se poser deux questions.

27 - Quel est le raccordement géométrique du réseau karstique étudié par rapport au contexte externe (notion d'altitude relative par rapport au niveau de base; 
éloignement du drain reconnu par rapport à l'événement fondateur, par exemple la vallée ; comblement partiel ou total de ce drain et sur quelle épaisseur)?

- Quel est le décalage temporel entre l'édification du réseau karstique (marqueur d'un événement géodynamique externe) et la pousse du spéléothème (notion de décalage lié à l'ouverture du système pour faciliter l'infiltration de l'eau, rôle des changements climatiques dans l'efficacité de la croissance des concrétions)?

Face à ces différents problèmes (analytique, spatial et temporel), des stratégies d'études et d'échantillonnages sont mises en place. Elles consistent généralement à multiplier les datations au sein d'un même réseau karstique, en cherchant en première approche les objets potentiellement les plus anciens (perchés dans le réseau ou bien à la base des coupes) avec le risque, parfois recherché, de dépasser la méthode (350 000 à 400000 ans). Dans la mesure du possible, plusieurs datations sont entreprises sur un même spéléothème afin de contrôler la succession chronologique conforme de celles-ci, voire de saisir les interruptions de croissance. Enfin, s'il est possible que plusieurs stalagmites encadrent un événement (avec la possibilité de le dater parfois finement), bien souvent les spéléothèmes de grottes ne proposent que des dates a minima (notion de scellement) ce qui implique des reconstitutions paléogéographiques limitées par une borne temporelle minimale. Conscients de ces limites, nous essayons ci- après de mesurer l'apport que peuvent constituer ces datations à la connaissance de l'évolution géomorphologique régionale.

\section{Apport à l'évolution du relief de l'Est du Bassin parisien}

L'apport des études karstiques et des datations U/Th des karsts de l'Est du Bassin parisien porte sur l'incision des vallées, le recul des couvertures et les variations climatiques.

\section{Sur l'incision des vallées}

31 C'est l'apport le plus fondamental. Qu'ils fonctionnent en pertes ou en émergences, les drains du karst s'étagent globalement en suivant l'incision des vallées desquels ils dépendent. Les spéléothèmes datés qui marquent l'abandon de ces karsts jalonnent, avec un certain décalage, les étapes de cette incision. Les datations obtenues sur le karst de la Moselle conduisent aujourd'hui à vieillir la date communément admise pour la capture de la Moselle de 250000 à plus de 300000 ans B.P. (Losson et Quinif, 2001).

Dans le Barrois, les karsts de Poissons, de la Cousances et du Rupt-du-Puits jalonnent l'incision du réseau hydrographique de la Marne et de ses affluents. Les datations obtenues montrent que cette incision s'est amorcée avant le Pléistocène moyen. Le caractère ancien du karst de Poissons, son comblement total par des matériaux issus du Crétacé et son altitude élevée militent en faveur d'un raccordement de ce karst au moins au Pléistocène ancien.

Les datations obtenues sur le karst de Cousances montrent qu'au Pléistocène récent, et en particulier à l'Eémien, la Marne est au plus à une dizaine de mètres au-dessus de ses écoulements actuels. Une telle datation impliquerait une vitesse d'incision maximale de l'ordre de 0,1 m par millénaire. Ceci s'accorde assez bien avec la découverte dans les terrasses perchées de l'Ornain d'une association d'industries et faunes situant les 
vallées de l'Ornain et de la Saulx vers + $25 \mathrm{~m}$ au début du Saalien (Guillaume, 1982, Harmand et al., 2002).

\section{Sur le recul des couvertures}

Le recul des couvertures est enregistré dans le karst à plusieurs échelles. À Trampot, au contact des Calcaires à Astartes et des calcaires de l'Oxfordien, on identifie des phases de recul de la couverture qui semblent être responsables de la détente lithostatique du réseau. Les spéléothèmes datés encadrent cette phase. Un plancher stalagmitique affecté par cette détente est daté 47500 à 49500 ans B.P. (fig. 5). Ceci permet de montrer que ce recul des Calcaires à Astartes (non chiffré en distance) a pu s'effectuer lors du dernier maximum glaciaire.

Dans le Barrois, les karsts s'égrènent du sud vers le nord, du plus ancien (Poissons Pléistocène ancien probable) au plus récent (Rupt-du-Puits - Pléistocène récent) et jalonnent le recul de la couverture crétacée sur les calcaires tithoniens. En effet, le karst de Poissons, le plus méridional, contient des restes de Crétacé démantelé alors qu'il en est éloigné aujourd'hui de plusieurs kilomètres. Au Rupt-du-Puits (le plus septentrional), la couverture est présente et mesure près de $30 \mathrm{~m}$ de puissance. Compte tenu de l'épaisseur de couverture qui devait exister au moment de la mise en place du karst de Poissons, le recul du front de la couverture crétacée, sur les calcaires tithoniens, a été évalué à une dizaine de kilomètres au cours de l'ensemble du Pléistocène (Jaillet, 2000).

\section{Sur les variations climatiques}

Les ensembles stalagmitiques édifiés au sein des systèmes karstiques présentés se sont mis en place sous des conditions climatiques particulières, jamais étudiées précisément dans le cadre strict de la Lorraine. En regroupant les 73 datations obtenues, on constate une organisation en groupes temporels présentés sur la figure 7, parallèlement à la courbe des rapports isotopiques de l'oxygène 18 (Petit et al., 1999).

Globalement, les concrétions poussent en stade chaud (stades isotopiques impairs), mais quelques dates se situent dans des stades isotopiques pairs (froid). Ainsi, les stades isotopiques 2 et 4 ont-ils vu la pousse de quelques concrétions dans le karst de Cousances. Aucun spéléothème n'a été reconnu dans le stade 5.5, chaud, de l'Eémien. Le stade 5.3 et le début du stade 3 sont riches en concrétions datées. Enfin le stade isotopique 1 , l'Holocène, concentre pratiquement tous les spéléothèmes datés dans le réseau du Rupt-du-Puits et un nombre important dans les grottes de Pierre-la-Treiche.

Ces résultats montrent (à la marge d'erreur près des datations $\mathrm{U} / \mathrm{Th}$ ) que la vision traditionnelle qui s'accorde d'une croissance des concrétions en périodes chaudes est peut-être à nuancer dans le cadre des karsts de l'Est du Bassin parisien. Les péjorations climatiques traditionnellement reconnues dans les karsts de montagnes (Maire, 1990 ; Delannoy, 1997) ou de hautes latitudes ne sont peut-être pas si marquées dans les karsts de bas-plateau. D'autres dates, des études plus fines, permettraient certainement de préciser cette approche qui dépasse le cadre de cette première synthèse. 


\section{Conclusion}

Les études menées sur les karsts associés aux grandes vallées de l'Est du Bassin parisien constituent un apport novateur à la connaissance de l'évolution géomorphologique et les datations U/Th entreprises sur ces karsts apportent un calage chronologique depuis le Pléistocène moyen. Dans les domaines de l'incision du réseau hydrographique, du recul des couvertures non carbonatées et des reconstitutions paléoclimatiques, ces études complètent et précisent les schémas d'évolution qui avaient été proposés à partir d'autres enregistreurs (formes et formations superficielles). La multiplication de ces datations sur ces mêmes karsts de Lorraine, mais aussi sur d'autres, situés dans des contextes morphoclimatiques proches (plateau de Haute-Saône, bordure ardennaise, Nivernais...) serait d'un apport certain à la connaissance des évolutions géomorphologiques de la France du Nord-Est. Cependant, le caractère discontinu de ces enregistreurs endokarstiques, le peu de spéléothèmes datables, leurs petites tailles, doivent inciter à la prudence sur l'utilisation disproportionnée de méthodes de datations, forcément destructrices, qui laisserait à l'avenir peu de concrétions utilisables. Des stratégies de prélèvement pourraient être proposées, qui permettraient d'identifier des cibles fiables, efficaces et correctement raccordées au contexte géomorphologique régional.

\section{BIBLIOGRAPHIE}

Les auteurs remercient David Carbon (GeoTer), Jean-Pierre Depaquis et Claude Herbillon pour leur aide lors des campagnes de prélèvement des échantillons. Richard Maire et André Weisrock ont bien voulu apporter une lecture constructive du manuscrit, contribuant ainsi à son amélioration. Qu'ils en soient remerciés.

AUDRA P. (2001). - L'organisation verticale des réseaux karstiques non confinés. Contrôle de la structure et du niveau de base. Actes de la $11^{\text {e }}$ rencontre d'octobre, Spéléo Club de Paris, Lisle-en-Rigault, 13-15.

BeAudoin J.P. (1974). - Phénomènes et dépôts karstiques du Barrois. Mém. Maîtrise, Univ. Nancy 2, $96 \mathrm{p}$.

BEAUdoIN J.P. (1989) - Karsts en Meuse, le travail des eaux souterraines en pays calcaires. Centre Départ. Doc. Pédagogique de la Meuse, Conseil général de la Meuse, 93 p. BUZZI P., CARCAUD N., KOENIG M.-P., WEISROCK A. (1993). - Morphodynamique fluviale holocène et établissements humains protohistoriques en fond de vallée de la Moselle à Crevéchamps (Lorraine méridionale). Revue Géogr. de l'Est, Tome 33, n 4, Nancy, 281-296.

CARCAUD N. (1990). - Etude préliminaire du comblement de fond de vallée de la Moselle à Vandières (Meurthe-et-Moselle). Revue Géogr. de l'Est, Tome 30, n 1, Nancy, 37-48. 
CORNUEL J. (1879). - Observations sur les puits naturels des calcaires portlandiens des départements de la Haute-Marne et de la Meuse et sur le minerai de fer qu'ils renferment. Bull. Soc. Géol. France, 3e série, t.1, 722-739.

DeLANNoy J.-J. (1997). - Recherches géomorphologiques sur les massifs karstiques du Vercors et de la Transversale de Ronda (Andalousie). Les apports morphogéniques du karst. Thèse d'Etat, Univ. Grenoble, Septentrion, 678 p.

DuRup de baleine A. (1989). - Le karst de la forêt de Hesse. Etude géomorphologique d'un karst vert en Lorraine occidentale. Mém. Maîtrise, Univ. Nancy 2, 286 p.

GAMEz P. (1977). - Etude géomorphologique d'un bassin versant karstique : les « bouillons » de Delut (Meuse). Mém. Maîtrise, Univ. Metz, CEGUM, 64 p.

GAMEZ P. (1985). - Karstologie lorraine. La spéléologie en Loraine, Spéléo- $L$ n 15, spécial congrès Féd. Fr. Spéléologie, Nancy, 35-46.

GAMEZ P. (1992). - Hydrologie et karstologie du bassin du Loison (Woëvre

septentrionale - Lorraine). Thèse, Univ. Metz, CEGUM, Mosella, t. 21, 1995, 397 p.

GAMEZ P., Losson B. (1998). - Premiers résultats de l'étude des remplissages dans le karst de Pierre-la-Treiche (54), l'entrée E du réseau Sainte-Reine. Mosella, Metz, t. 23, nº 3-4, 41-59.

GAMEZ P., WEHRLI A., FIZAINE J.-P., SCAPOLI J. (1995). - L'implication du karst dans la capture de la Moselle. Revue Géogr. de l'Est, t. 35, n³-4, Nancy, 297-308.

Guillaume C. (1982). - Stations de surface du Paléolithique inférieur et moyen de Lorraine. Bull. de I'Assoc. Fr. pour l'Etude du Quaternaire, n 2-3, 135-146.

HARMAnd D. (1992). - Histoire de la vallée de la Meuse lorraine. Thèse Univ. Nancy 2, Presses univ., Nancy, $146 \mathrm{p}$.

HARMAND D., FAUVEL P.-J., JAILLET S., LE ROUX J.,ALLOUC J., BRULHET J. ET BROCANDEL M. (2002). — Incisions ante- et post-

capture dans les vallées de l'Ornain et de la Saulx, Revue géogr. de l'Est, t. 42, n 4 (en ce volume).

HARMAND D., WEISROCK A., GAMEZ P., LE ROUX J., OCCHIETTI S., DESHAIES M., BONNEFONT J.-C., SARY M. (1995). Nouvelles données relatives à la capture de la Moselle. Revue Géogr. de l'Est, t. 35, n 3-4, Nancy, 321-343.

JAILLET S. (2000). - Un karst couvert de bas-plateau : le Barrois. Structurefonctionnement-évolution. Thèse, Univ. Bordeaux 3, 710 p.

JAILLET S., GAMEZ P (1995). - Observations morphologiques sur le géosystème du Rupt-du-Puits. Karstologia, 26, 27-38.

JAILLET S., PONS-BRANCHU E., BRULHET J. et HAMELIN B. (à paraître). - Karstification as a geomorphological witness of river incision: the karst of Cousance and the Marne Valley (Eastern Paris Basin). Terra Nova, soumis.

LejeUne O., MARRE A., DEVoS A., LAURAin M. et BRULhet J. (2002). - Les phases d'incision de la vallée de la Marne entre Joinville et Vitry-le-François. Revue géogr. de l'Est, t. 42, nº 4 (en ce volume).

LE ROUX J., HARMAND D. (1998). - Contrôle morphostructural de l'histoire d'un réseau

hydrographique : le site de la capture de la Moselle. Gemodinamica Acta, vol. 11, nº 4, 149-162. 
Losson B. (2000). - Modalités des défluviations partielles souterraines de la Moselle avant sa capture. Bull. Info. Géol. Bassin Paris, vol. 37, n 3, 15-22.

LOSSON B. (2001). - Quelques aspects de la karstification du plateau de Haye (Lorraine, France). Actes $3^{\mathrm{e}}$ Journée Spéléologie Scientifique, Han-sur-Lesse, Regards, n 41, 26-28.

Losson B. et QUINIF Y. (2001). - La capture de la Moselle ; nouvelles données chronologiques par datations U/Th sur spéléothèmes. Karstologia, 37, 29-40.

MAIRE R. (1990). - La haute montagne calcaire. Karsts, Cavités, Remplissages,

Quaternaire, Paléoclimats. Thèse d'Etat, Karstologia-mémoires n 3, 731 p.

PETIT J.R., JOUZEL J., RAYNAUD D., BARKOV N.I., BARNOLA J.-M., BASILE I., BENDER M., CHAPPELLAZ J., DAVIS M., DELAYGUE G., DELMOTTE M., KOTLYAKOV V.M., LEGRAND M., LIPENKOV V.Y., LORIUS C., PÉPIN L., RITZ C., SALTZMAN E. et STIEVENARD M. (1999). - Climate and atmospheric history of the past 420,000 years from the Vostok ice core, Antarctica. Nature, 399, 429-436.

PISSART A., HARMAND D., KROOK L. (1997). — L'évolution de la Meuse de Toul à Maastricht depuis le Miocène : corrélations chronologiques et traces des captures de la Meuse lorraine d'après les minéraux denses. Géogr. Phys. et Quaternaire, vol. 51, n 3, Montréal, 267-284.

Pons-BRANCHU E. (2001). - Datations hautes-résolutions de spéléothèmes (230Th/ $234 U$ et 226Ra/226U). Application aux reconstitutions environnementales autour des sites du Gard et de Meuse / Haute-Marne. Thèse Univ. Aix-Marseille, ANDRA, 225 p. QUINIF Y. (1989). - La datation uranium-thorium. Spéléochronos, n 1, CERAK, Mons, pp. 3-21. QUINIF Y. (1994). - Hit 15 : la plus belle synthèse régionale : le karst de Couvin. TOP 15 des phénomènes karstiques wallons. Lapiaz, hors-série, 43-47.

QUINIF Y. (1998). - Dissipation d'énergie et adaptabilité dans les systèmes karstiques. Karstologia, 31, 1-11.

WEISROCK A. (1993). - Le remplissage tardiglaciaire et holocène des vallées lorraines. L'eau, la Terre et les Hommes : au fil de l'eau. Hommage à R. Frécaut. Presses universitaires de Nancy, 303-309.

\section{NOTES}

1. Les thèses de Jaillet (2000) et Losson (en cours) ont été mises en œuvre sous la direction de Patrice Gamez $(\dagger)$.

2. ANDRA : Agence Nationale pour la gestion des Déchets Radioactifs.

3. Spectrométrie 頤. Cette méthode est basée sur la mesure de l'activité de tous les isotopes d'Uranium. Elle nécessite généralement une quantité importante de calcite.

4. TIMS : Thermal Ionization Mass spectrometry. Cette méthode de spectrométrie de masse est basée sur la mesure de la masse des différents isotopes de l'Uranium. Elle peut être mise en œuvre avec des quantités d'échantillons bien plus petites. 


\section{RÉSUMÉS}

Dans l'Est du Bassin parisien, la karstification s'exprime selon deux modes essentiels, soit au contact d'une couverture argilo-sableuse sur les plateaux calcaires, soit au contact d'une nappe alluviale dans les fonds de vallée. Dans tous les cas, l'évolution de ces karsts suit l'incision des vallées. On présente ici trois sites karstiques majeurs (Pierre-la-Treiche, Trampot et le Barrois), inféodés à trois grandes vallées de l'Est du Bassin parisien (la Moselle, la Meuse et la Marne) recoupant trois grandes masses carbonatées (Bajocien, Oxfordien, Tithonien). Dans ces karsts, 84 datations ont été entreprises par la méthode U/Th soit en alpha, soit en TIMS. La synthèse de ces dates confirme l'apport que constitue le karst dans les reconstitutions paléoenvironnementales en particulier dans le domaine de l'incision du réseau hydrographique, du recul des couvertures non carbonatées et des reconstitutions paléoclimatiques.

In the eastern part of the Paris Basin, karstification occurs, essentially in two modes, one at the contact of a sandy-clayey cover on limestone plateaus, the other beneath alluvium in the floors of valleys. In every case, evolution of the karsts follows incision of the valleys. In this paper, three major karstic systems are presented (Pierre-la-Treiche, Trampot and the Barrois), associated with three large valleys (the Moselle, the Meuse and the Marne), cutting three large limestone masses (Bajocien, Oxfordien, Tithonien). In these karsts, 84 dates were obtained using the U/Th method (alpha, TIMS). These dates confirm the contribution of the karst to palaeoenvironmental reconstruction in the fields of river system incision, retreat of the noncarbonate cover and palaeoclimatic reconstruction.

Im Osten des Pariser Beckens erscheint die Verkarstung in zwei wesentlichen Formen - in Kontakt mit einer lehmig-sandigen Decke auf den Kalkplateaus oder in Kontakt mit einer alluvialen Schicht in den Talgründen. In allen Fällen folgt die Entwicklung dieser Karste dem Einschneiden der Täler. Hier werden drei größere Karstbereiche vorgestellt (Pierre-la-Treiche, Trampot und das Barrois), die drei grossen Tälern (Mosel, Maas und Marne) zugehören und drei grosse Kalkmassive (Bajocien, Oxfordien, Tithonien) anschneiden. In diesen Karstgebieten wurden 84 Datierungen nach der Methode U/Th in alpha oder in TIMS vorgenommen. Die Synthese dieser Daten bestätigt den Beitrag, den der Karst für die paläogeographische Rekonstruierung liefert, insbesondere im Bereich des Einschneidens des hydrographischen Netzes, des Rückweichens der nichtkalkigen Deckschichten und paläoklimatischer Rekonstruktionen.

\section{INDEX}

Keywords : karst, Paris Basin., speleothems, U/Th datings, valley incision

Schlüsselwörter : Datierungen, Höhlenkunde, Karst, Pariser Becken., Taleinschneiden, U/Th

Mots-clés : Bassin parisien., datations U/Th, incision de vallées, karst, spéléothèmes 


\section{AUTEURS}

\section{STÉPHANE JAILLET}

UMR CNRS 7566 G2R « Géologie et Gestion des Ressources Minérales et Énergétiques », Université Henri Poincaré - Nancy 1, BP 239, 54506 Vandœuvre-lès-Nancy - Mel : Stephane.Jaillet@g2r.uhpnancy.fr

\section{BENOÎT LOSSON}

CEGUM - Centre d'Etudes Géographiques de l'Université de Metz, Île du Saulcy, 57045 Metz cedex.

\section{JACQUES BRULHET}

ANDRA - Agence Nationale pour la gestion des Déchets RadioActifs, Direction Scientifique, 1/7, rue Jean-Monnet, 92298 Châtenay-Malabry.

\section{JEANNINE CORBONNOIS}

CEGUM - Centre d'Etudes Géographiques de l'Université de Metz, île du Saulcy, 57045 Metz cedex.

\section{BRUNO HAMELIN}

UMR CNRS 6635 CEREGE « Geosciences de l'environnement », Europole de l'Arbois, BP 80, 13545 Aix-en-Provence Cedex 4.

\section{EDWIGE PONS-BRANCHU}

ANDRA - Agence Nationale pour la gestion des Déchets RadioActifs, Direction Scientifique, 1/7, rue Jean-Monnet, 92298 Châtenay-Malabry.UMR CNRS 6635 CEREGE « Geosciences de l'environnement », Europole de l'Arbois, BP 80, 13545 Aix-en-Provence Cedex 4.

\section{YVES QUINIF}

CERAK - Centre d'Études et de Recherches appliquées au karst, Faculté polytechnique de Mons, rue de Houdain 9, 7000 Mons (Belgique). 